\title{
Effect of Side Surface Orientation on the Mechanical Properties of Silicon Nanowires: A Molecular Dynamics Study
}

\author{
Xiao Ru Zhuo ${ }^{1}$ and Hyeon Gyu Beom ${ }^{2, *}$ \\ 1 College of Mechanics and Materials, Hohai University, Nanjing 210098, China; xrzhuo@hhu.edu.cn \\ 2 Department of Mechanical Engineering, Inha University, Incheon 402-751, Korea \\ * Correspondence: hgbeom@inha.ac.kr; Tel.: +82-32-860-7310
}

Received: 20 January 2019; Accepted: 12 February 2019; Published: 18 February 2019

\begin{abstract}
We investigated the mechanical properties of $<100>$-oriented square cross-sectional silicon nanowires under tension and compression, with a focus on the effect of side surface orientation. Two types of silicon nanowires (i.e., nanowires with four $\{100\}$ side surfaces and those with four $\{110\}$ side surfaces) were simulated by molecular dynamics simulations at a temperature of $300 \mathrm{~K}$. The deformation mechanism exhibited no dependence on the side surface orientation, while the tensile strength and compressive strength did. Brittle cleavage was observed under tension, whereas dislocation nucleation was witnessed under compression. Silicon nanowires with $\{100\}$ side surfaces had a lower tensile strength but higher compressive strength. The effect of side surface orientation became stronger as the nanowire width decreased. The obtained results may provide some insight into the design of silicon-based nano-devices.
\end{abstract}

Keywords: molecular dynamics simulation; silicon nanowires; side surface orientation effect; tensile strength; compressive strength

\section{Introduction}

Silicon nanowires have attracted tremendous interest from worldwide scientific communities and various industries, because of their extraordinary optical [1], electrical [2], thermoelectric [3], piezoelectric [4], and mechanical [5] properties. As an essential building block in nanotechnology, silicon nanowires have been widely applied in nanoelectromechanical systems, such as field effect transistors, nanoresonators, nanosensors, light-emitting diodes, logic gates, and battery anodes. In these devices, silicon nanowires are often under extreme loading conditions. For example, in the fabrication process of a lithium battery, the volume of silicon nanowires can change about $400 \%$ because of the Li insertion [6]; in the silicon-based field effect transistors, silicon nanowires can be strained more than $12 \%$ [7]. Once the applied strain exceeds a critical value, dislocations or cracks may initiate in silicon nanowires, which can degrade the function of these silicon-based devices. Therefore, to maintain the reliability of these devices, it is essential to gain a thorough knowledge of the mechanical properties of silicon nanowires.

When the sizes of materials reduce to the nanoscale, their mechanical properties may become sensitive to many factors, such as size, shape, surface roughness, native oxide layer, and loading strain rate. The influence of these factors on the mechanical properties of silicon nanowires has been intensively studied through experiments and atomistic simulations, and some interesting phenomena have been observed. For example, the manner in which silicon nanowire fractures may change from brittle to ductile once its diameter is less than a critical value [5,8,9]; silicon nanowires with a circular cross-section are stronger than those with a square cross-section under tension, but the reverse 
happens under compression [10]; the native oxide layer can reduce the Young's modulus of silicon nanowires [11,12]; the yield strength of silicon nanowires decreases with increasing strain rate and temperature [13]; the yield mechanism of silicon nanowires under torsional loading changes from dislocation activities to crystal-to-amorphous transition when the diameter decreases to a critical value [14].

Many growth methods, including chemical vapor deposition [15], laser ablation [16], and thermal evaporation [17], have been developed to synthesize nanowires. Using different methods, silicon nanowires with different growth directions can be synthesized and silicon nanowires with the same growth direction can have different side surfaces [18]. The side surface orientation plays a dominant role in determining the operant mode of inelastic deformation in both $<100>$ and $<110>$ face-centered cubic nanowires [19]. However, its effect on the mechanical properties of silicon nanowires is still not clear.

In this work, we aim to investigate the influence of side surface orientation on the mechanical properties of silicon nanowires. To this end, molecular dynamics (MD) simulations of the deformation behavior of silicon nanowires under tensile and compressive loading were conducted. MD simulations can provide insight into detailed atomic deformation mechanisms [20], which are not available from experiments.

\section{Simulation Method}

Two types of silicon nanowires were simulated in this work, as schematically illustrated in Figure 1. Nanowires with a $<100>$ longitudinal axis and four $\{100\}$ side surfaces were called $<100>/\{100\}$ nanowire. Similarly, those with a $<100>$ longitudinal axis and four $\{110\}$ side surfaces were named $<100>/\{110\}$ nanowire. The aspect ratio (length/width) of all nanowires was fixed to be three. A similar aspect ratio has been widely used in previous work $[10,21,22]$. In order to investigate the size effect, five widths $(w=2.7,3.5,4.6,5.7,7.7 \mathrm{~nm})$ were considered.

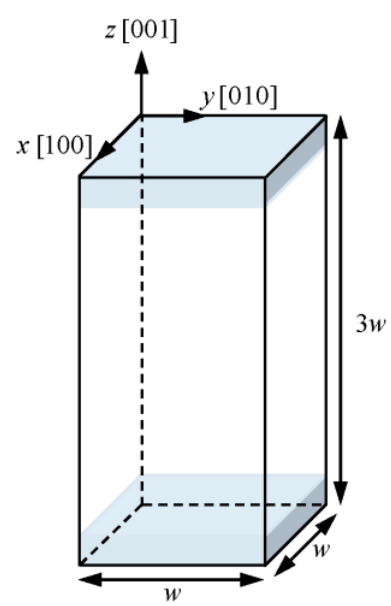

$<100>/\{100\}$ nanowire

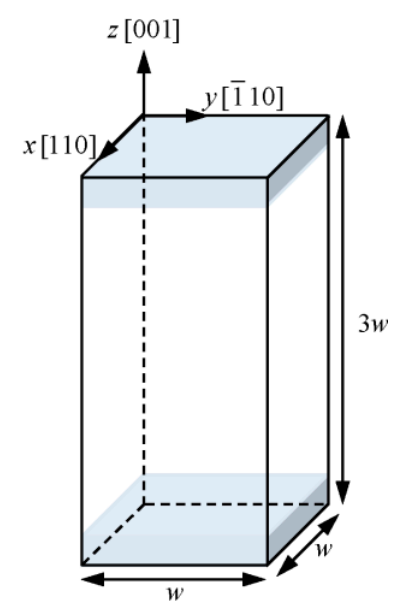

$<100>/\{110\}$ nanowire

Figure 1. Geometry of the atomic model for silicon nanowires. The shaded areas represent the frozen atoms, and the transparent areas denote the active atoms.

A reliable potential model is a key to maintaining the accuracy of simulation results [23]. Although the ab initio simulations are the most reliable computational techniques, they are not suitable for modeling large systems due to the heavy calculation burden. Many empirical interatomic potentials have been developed to describe the Si-Si interaction, including the Tersoff potential [24], the Stillinger-Weber (SW) potential [25], the environment-dependent interatomic potential (EDIP) [26], and the modified embedded atom method (MEAM) potential [27]. Among these empirical potentials, the MEAM potential is most reliable [8,28]. Therefore, the MEAM potential was utilized and the pair cutoff was set to be $0.6 \mathrm{~nm}$, consistent with previous work [8,23]. 
MD simulations were conducted using the open source molecular dynamics program, Large-scale Atomic/Molecular Massively Parallel Simulator (LAMMPS) [29], and the atomic configurations were visualized by the visualization software AtomEye [30]. In all simulations, periodic boundary conditions were not used and the time step was set to be $1 \mathrm{fs}$. The simulation system was divided into three regions: Top region, the active region, and bottom region. The top and bottom regions, which both contain six atomic layers, are shaded in gray in Figure 1. The whole simulation model was first relaxed to the minimum energy state using the conjugate gradient method. After relaxation, the top and bottom regions were frozen and the active region was equilibrated for $50 \mathrm{ps}$ in the NVT ensemble at $300 \mathrm{~K}$. Subsequently, to apply a tensile/compressive strain, the bottom region remained fixed and the top region was moved along the longitudinal axis with a constant velocity, corresponding to a strain rate of $10^{9} \mathrm{~s}^{-1}$. This strain rate was chosen since the deformation behavior of nanowires is independent on the strain rate once it is smaller than about $10^{10} \mathrm{~s}^{-1}[31,32]$.

The stress tensor of silicon nanowires was calculated using the virial stress [33]. The atomic-level virial stress has been demonstrated to be equivalent to the continuum-level Cauchy stress [34]. The strain was calculated according to the definition of engineering strain.

\section{Results and Discussions}

\subsection{Stress-Strain Curves}

In order to monitor the response of silicon nanowires to the applied tensile and compressive loading, the stress-strain curves were plotted. The stress-strain curves of silicon nanowires showed similar trends regardless of the nanowire width. Therefore, for clarity, only those for the nanowire with a width of $7.7 \mathrm{~nm}$ were shown in Figure 2. Here, the stress was the absolute value of the average atomic stress $\left(\sigma_{33}\right)$ over the entire volume of the active region of the simulation model and the strain was the absolute value of the applied strain. Initially, both types of silicon nanowires responded linearly to the applied strain and the Young's modulus was calculated from this stage. The obtained values fell into the range of 120-130 GPa, in close agreement with previous simulation results [35] and experimental data [36]. No obvious size or surface orientation effects on the Young's modulus were observed, in consistency with a previous simulation result that the Young's modulus of silicon nanowires exhibited negligible size-dependence when their diameter was larger than about $3 \mathrm{~nm}$ [32].

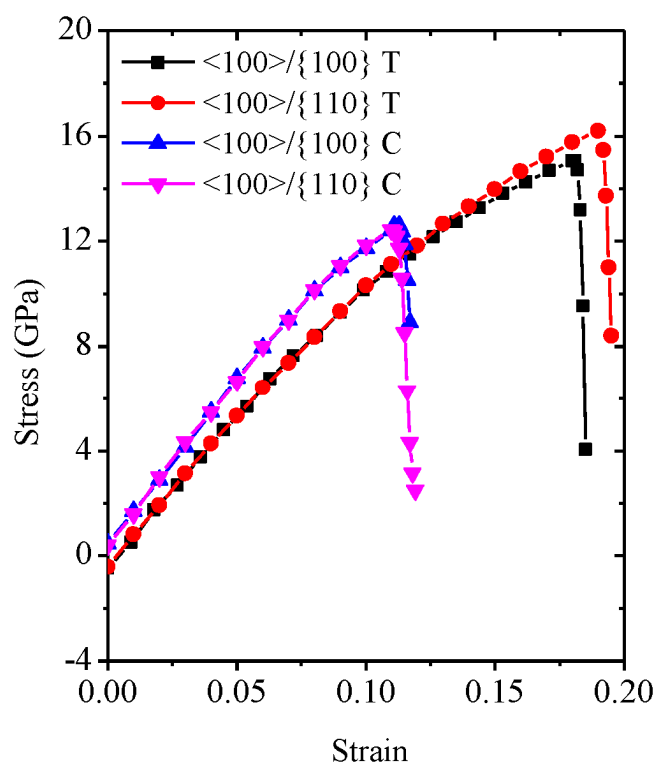

Figure 2. Stress-strain curves of the silicon nanowire with a width of $7.7 \mathrm{~nm}$. The character " $\mathrm{T}$ " and "C" designate "tension" and "compression", respectively. Both compressive stress and strain are taken as positive value. 
With the applied strain increasing to a large value, the stress-strain curves became somewhat nonlinear and this nonlinear trend became more and more obvious with the increase of strain. Moreover, tensile deformations exhibited stronger nonlinearity than compressive deformations. A similar nonlinear response has been reported in previous studies on silicon nanofilms [23] and nanowires [8,10,28], and it was attributed to their nanoscale size [23]. After reaching its peak value, the stress experienced a sharp decline, which may have been associated with crack or dislocation nucleation. To find the exact reason, detailed deformation mechanism of silicon nanowires should be analyzed.

\subsection{Tensile Deformation Mechanism}

Snapshots depicting the evolution of the atomic configuration of silicon nanowires were captured to demonstrate their deformation mechanism under tension. The nanowire width and side surface orientation did not affect the tensile deformation mechanism of silicon nanowires. Hence, only snapshots for the $<100>/\{110\}$ silicon nanowire with a width of $7.7 \mathrm{~nm}$ are shown in Figure 3 . Silicon nanowires deform elastically and a crack nucleates on their side surface when the critical tensile strain is reached. Crack nucleation led to a sharp decline in tensile stress, observed in Figure 2. On further strain, the nucleated crack propagated along the (001) plane, as demonstrated in Figure 3c. When the tensile strain reached a critical value, brittle cleavage occurred to silicon nanowires, as shown in Figure 3d.

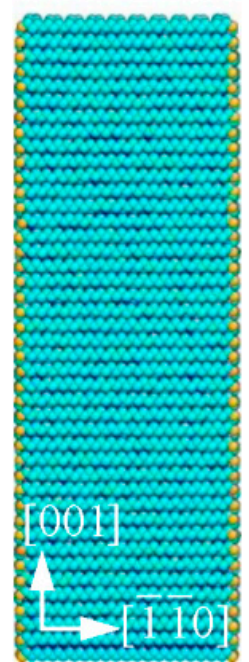

(a)

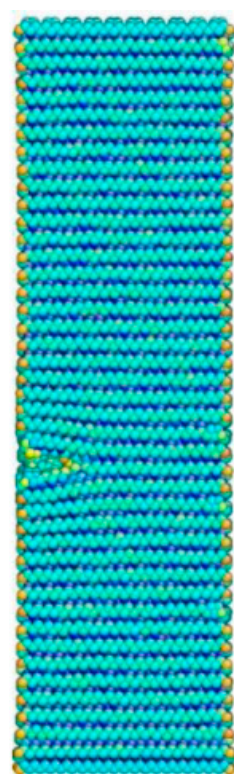

(b)



(c)

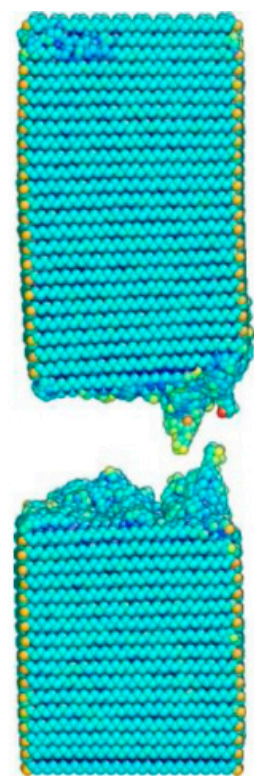

(d)

Figure 3. Atomistic configurations of the $<100>/\{110\}$ silicon nanowire with width of $7.7 \mathrm{~nm}$ at different tensile strain levels. Atoms are colored according to their potential energy, with blue indicating low value and red representing high value. (a) $\varepsilon=0$, (b) $\varepsilon=0.189$, (c) $\varepsilon=0.191$ and (d) $\varepsilon=0.196$.

The brittle fracture of silicon nanowires observed in this work is consistent with some previous simulation results [21,22] and experimental observations [37]. However, in some other simulation work [10,38,39] and experimental studies [40], ductile fracture and brittle-to-ductile transition were observed. The difference in the potential model may induce the difference in the deformation mechanism observed in atomistic simulations. Some empirical potential models, such as SW potential and Tersoff potential, can result in a non-physical plastic mechanism [21,22,28]. Due to the imperfection of the fabrication process, the silicon nanowires used in experiments are often covered with an amorphous layer [37]. In addition, many other imperfections, such as vacancies and dislocations, may exist in silicon nanowires. These imperfections may make it easier for a dislocation to nucleate and 
move in silicon nanowires, resulting in the ductile behavior. Once the amorphous layer is crystallized, silicon nanowires would behave in a brittle manner [37].

\subsection{Compressive Deformation Mechanism}

The deformation mechanism of silicon nanowires under compression is not influenced by nanowire width or side surface orientation. The structural evolution of the $<100>/\{100\}$ silicon nanowire under compression is exhibited in Figure 4. In this figure, all atoms are colored according to the local von Mises shear strain invariant [41], with blue indicating a low value and red representing a high value. For clarity, only atoms with a local von Mises shear strain invariant larger than 0.2 are shown. Initially, silicon nanowires responded elastically to the applied compressive strain. Once the compressive strain reached a critical value, the dislocations nucleated from the intersection of two side surfaces, as shown in Figure $4 b$,f. The nucleated dislocations had a Burger vector of $1 / 2<011>$. The dislocation nucleation resulted in a sharp drop in compressive stress, shown in Figure 2. With the increase of compressive strain, the nucleated dislocations propagated along the $\{011\}$ plane (Figure $4 c, g$ ) and a step were created on one side surface eventually (Figure $4 \mathrm{~d}, \mathrm{~h}$ ).

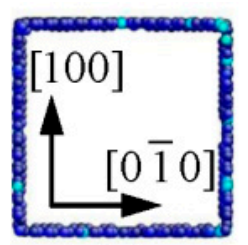

(a)

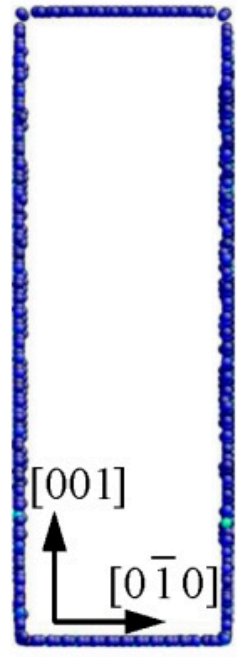

(e)

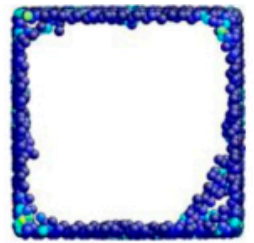

(b)

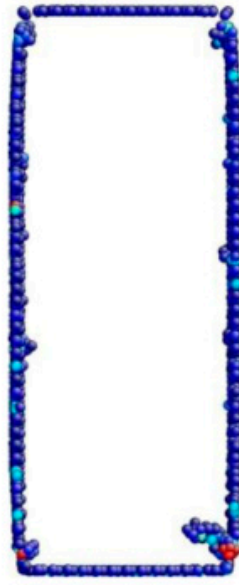

(f)



(c)

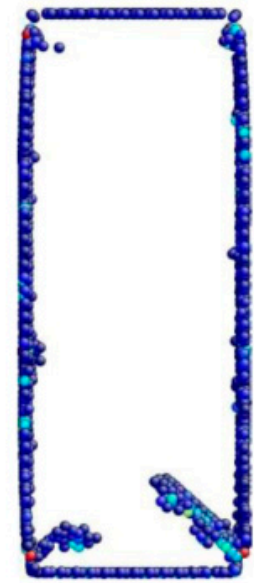

(g)

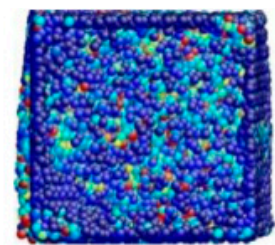

(d)



(h)

Figure 4. Atomistic configurations of the $<100>/\{100\}$ silicon nanowire with width of $7.7 \mathrm{~nm}$ at different compressive strain levels. Atoms are colored according to the local von Mises shear strain invariant [41], with blue indicating low value and red representing high value. (a) $\varepsilon=0$, (b) $\varepsilon=0.111$, (c) $\varepsilon=0.113$, (d) $\varepsilon=0.116$, (e) $\varepsilon=0,(\mathbf{f}) \varepsilon=0.111,(\mathbf{g}) \varepsilon=0.113$ and (h) $\varepsilon=0.116$. For clarity, only atoms with local von Mises shear strain invariant larger than 0.2 are shown.

The plastic deformation of silicon nanowires observed in this study correlated well with a previous experimental observation that the uniaxial compressive deformation of silicon nanowires changes from brittle to ductile once their diameters are below a critical value (310-400 $\mathrm{nm}$ ) [9]. However, the slip planes observed in our simulations were $\{011\}$ planes, different from the $\{111\}$ planes reported from experiments. The activation of the unexpected $\{011\}$ slip planes has been observed in previous MD simulations $[10,21,22]$, and their occurrence was demonstrated to be an indirect consequence of the small dimensions of silicon nanowires [21]. 


\subsection{Tensile/Compressive Strength}

The tensile strength and compressive strength of both types of silicon nanowires were extracted from the stress-strain curves, as shown in Figure 5, as a function of nanowire width. The tensile strength was in the range of 13.5 to $16.2 \mathrm{GPa}$, while the compressive strength ranged from 10.2 to $12.6 \mathrm{GPa}$. Similar results have been reported in previous work $[8,28]$. Regardless of the type of silicon nanowires, the tensile/compressive strength showed similar trends with the variation of nanowire width. Specifically, both the tensile strength and compressive strength increased with increasing nanowire width, which was in close agreement with previous simulation results $[8,13,38,39]$. The surface atoms of silicon nanowires are under-coordinated and they have higher potential energy than atoms inside nanowires, which makes the surfaces the weak sites for dislocations or cracks to nucleate. As the nanowire width decreased, the surface-to-volume ratio increased and thus the tensile/compressive strength reduced. However, the tensile/compressive strength was observed to increase with reducing nanowire size in experiments [42]. The contradiction between simulation results and experimental observations could be explained by the imperfection of the silicon nanowires used in the experiments. Defects are inevitably introduced into silicon nanowires during the fabrication process, and these defects are the potential nucleation sites for cracks and dislocations. With the decrease of nanowire width, the defect population reduces, inducing an increase in the tensile/compressive strength.

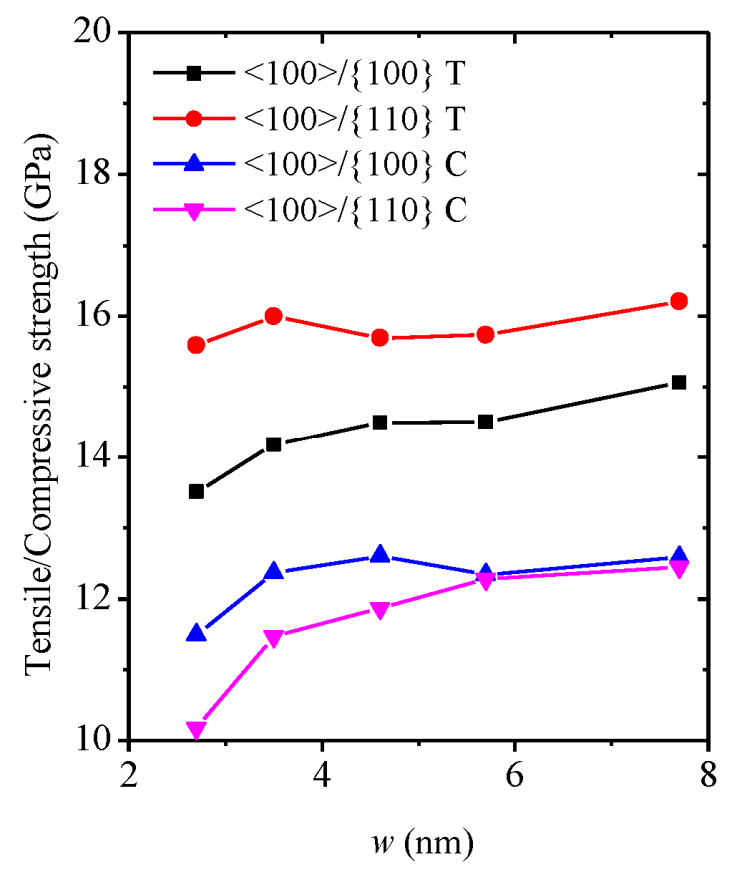

Figure 5. Tensile/compressive strength of silicon nanowires as a function of nanowire width $w$. The character " $\mathrm{T}$ " and " $\mathrm{C}$ " designate "tension" and "compression", respectively.

Side surface orientation exerts a strong influence on the tensile strength and compressive strength of silicon nanowires. Similar side surface orientation effect was observed in the tungsten nanowires [43]. Under tensile loading, the $<100>/\{110\}$ nanowires were stronger than the $<100>/\{100\}$ nanowires; whereas under compressive loading the reverse is true. The under-coordination of surface atoms can result in surface stress in materials. In bulk materials, the role of surface stress is insignificant due to the small surface-to-volume ratio. However, in nanowires which are characterized by a large surface-to-volume ratio, surface stress becomes important. Surface stress can give rise to many interesting phenomena, such as the tensile-compressive yield strength asymmetry in metal nanowires [44] and the phase transformation in gold nanowires [45]. It is conjectured that the effect of side surface orientation may originate from surface stress. The effect of side surface orientation was affected to some extent by the nanowire width, as shown in Figure 5. With the increase of the 
nanowire width, the side surface orientation effect seemed to become weak. When the nanowire width scaled down, the surface-to-volume ratio increased, and the role that surfaces play in the mechanical properties of nanowires became more important. Hence, the side surface orientation effect was stronger in smaller nanowires.

\section{Conclusions}

The deformation behaviors of square cross-sectional $<100>$-oriented silicon nanowires with $\{100\}$ and $\{110\}$ sides surfaces were simulated by using MD simulations. The side surface orientation did not affect the deformation mechanism, but the loading direction did. Under tensile strain, cracks nucleated on the side surfaces, which induced the brittle cleavage; while under compressive strain, dislocations nucleated on the side surfaces, which resulted in the slip along the $\{011\}$ plane. Both the tensile strength and compressive strength were strongly influenced by the side surface orientation. Silicon nanowires with $\{110\}$ side surfaces were stronger than those with $\{100\}$ side surfaces under tension, but the reverse was true under compression. The effect of side surface orientation was stronger in smaller nanowires, since the side surfaces played an increasingly important role in the mechanical properties of silicon nanowires with the increase of the surface-to-volume ratio.

Author Contributions: X.R.Z., and H.G.B. conceived and designed the study; X.R.Z. performed the simulations; X.R.Z. and H.G.B. analyzed the results and wrote the paper.

Funding: This research was supported by the Basic Science Research Program through the National Research Foundation of Korea (NRF), funded by the Ministry of Education, Republic of Korea (Grant No. NRF-2015R 1D 1A 1A01056866). Zhuo was supported by the Fundamental Research Funds for the Central Universities (Grant No. 2019B16414) and the China Postdoctoral Science Foundation (Grant No. 2018M632214).

Conflicts of Interest: The authors declare no conflict of interest.

\section{References}

1. Holmes, J.D.; Johnston, K.P.; Doty, R.C.; Korgel, B.A. Control of thickness and orientation of solution-grown silicon nanowires. Science 2000, 287, 1471-1473. [CrossRef]

2. Cui, Y.; Duan, X.; Hu, J.; Lieber, C.M. Doping and electrical transport in silicon nanowires. J. Phys. Chem. B 2000, 104, 5213-5216. [CrossRef]

3. Boukai, A.I.; Bunimovich, Y.; Tahir-Kheli, J.; Yu, J.-K.; Goddard Iii, W.A.; Heath, J.R. Silicon nanowires as efficient thermoelectric materials. Nature 2008, 451, 168-171. [CrossRef] [PubMed]

4. He, R.; Yang, P. Giant piezoresistance effect in silicon nanowires. Nat. Nanotechnol. 2006, 1, 42. [CrossRef] [PubMed]

5. Zhuo, X.R.; Beom, H.G. Atomistic study of the bending properties of silicon nanowires. Comput. Mater. Sci. 2018, 152, 331-336. [CrossRef]

6. Chan, C.K.; Peng, H.; Liu, G.; McIlwrath, K.; Zhang, X.F.; Huggins, R.A.; Cui, Y. High-performance lithium battery anodes using silicon nanowires. Nat. Nanotechnol. 2008, 3, 31-35. [CrossRef] [PubMed]

7. Lu, K.-C.; Wu, W.-W.; Wu, H.-W.; Tanner, C.M.; Chang, J.P.; Chen, L.J.; Tu, K. In situ control of atomic-scale Si layer with huge strain in the nanoheterostructure $\mathrm{NiSi} / \mathrm{Si} / \mathrm{NiSi}$ through point contact reaction. Nano Lett. 2007, 7, 2389-2394. [CrossRef]

8. Kang, K.; Cai, W. Size and temperature effects on the fracture mechanisms of silicon nanowires: Molecular dynamics simulations. Int. J. Plast. 2010, 26, 1387-1401. [CrossRef]

9. Östlund, F.; Rzepiejewska-Malyska, K.; Leifer, K.; Hale, L.M.; Tang, Y.; Ballarini, R.; Gerberich, W.W.; Michler, J. Brittle-to-ductile transition in uniaxial compression of silicon pillars at room temperature. Adv. Funct. Mater. 2009, 19, 2439-2444. [CrossRef]

10. Yang, Z.; Lu, Z.; Zhao, Y.-P. Shape effects on the yield stress and deformation of silicon nanowires: A molecular dynamics simulation. J. Appl. Phys. 2009, 106, 023537. [CrossRef]

11. Ilinov, A.; Kuronen, A. Atomistic modeling of bending properties of oxidized silicon nanowires. J. Appl. Phys. 2014, 115, 104305. [CrossRef]

12. Jing, Y.; Meng, Q. Molecular dynamics simulations of the mechanical properties of crystalline/amorphous silicon core/shell nanowires. Phys. B Condens. Matter 2010, 405, 2413-2417. [CrossRef] 
13. Jing, Y.; Meng, Q.; Zhao, W. Molecular dynamics simulations of the tensile and melting behaviours of silicon nanowires. Phys. E Low-Dimens. Syst. Nanostruct. 2009, 41, 685-689. [CrossRef]

14. Zhuo, X.R.; Beom, H.G. Size-dependent torsional deformation of silicon nanowires. Mater. Lett. 2018, 213, 48-50. [CrossRef]

15. Kamins, T.; Stanley Williams, R.; Basile, D.; Hesjedal, T.; Harris, J. Ti-catalyzed Si nanowires by chemical vapor deposition: Microscopy and growth mechanisms. J. Appl. Phys. 2001, 89, 1008-1016. [CrossRef]

16. Morales, A.M.; Lieber, C.M. A laser ablation method for the synthesis of crystalline semiconductor nanowires. Science 1998, 279, 208-211. [CrossRef] [PubMed]

17. Yu, D.; Bai, Z.; Ding, Y.; Hang, Q.; Zhang, H.; Wang, J.; Zou, Y.; Qian, W.; Xiong, G.; Zhou, H. Nanoscale silicon wires synthesized using simple physical evaporation. Appl. Phys. Lett. 1998, 72, 3458-3460. [CrossRef]

18. Li, C.P.; Lee, C.S.; Ma, X.L.; Wang, N.; Zhang, R.Q.; Lee, S.T. Growth direction and cross-sectional study of silicon nanowires. Adv. Mater. 2003, 15, 607-609. [CrossRef]

19. Park, H.S.; Gall, K.; Zimmerman, J.A. Deformation of FCC nanowires by twinning and slip. J. Mech. Phys. Solids 2006, 54, 1862-1881. [CrossRef]

20. Tang, D.-M.; Ren, C.-L.; Wang, M.-S.; Wei, X.; Kawamoto, N.; Liu, C.; Bando, Y.; Mitome, M.; Fukata, N.; Golberg, D. Mechanical properties of Si nanowires as revealed by in situ transmission electron microscopy and molecular dynamics simulations. Nano Lett. 2012, 12, 1898-1904. [CrossRef] [PubMed]

21. Guénolé, J.; Brochard, S.; Godet, J. Unexpected slip mechanism induced by the reduced dimensions in silicon nanostructures: Atomistic study. Acta Mater. 2011, 59, 7464-7472. [CrossRef]

22. Guénolé, J.; Godet, J.; Brochard, S. Deformation of silicon nanowires studied by molecular dynamics simulations. Model. Simul. Mater. Sci. Eng. 2011, 19, 074003. [CrossRef]

23. Zhuo, X.R.; Beom, H.G. Size-dependent fracture properties of cracked silicon nanofilms. Mater. Sci. Eng. A 2015, 636, 470-475. [CrossRef]

24. Tersoff, J. Empirical interatomic potential for silicon with improved elastic properties. Phys. Rev. B 1988, 38, 9902. [CrossRef]

25. Tersoff, J. Modeling solid-state chemistry: Interatomic potentials for multicomponent systems. Phys. Rev. B 1989, 39, 5566. [CrossRef]

26. Bazant, M.Z.; Kaxiras, E.; Justo, J.F. Environment-dependent interatomic potential for bulk silicon. Phys. Rev. B 1997, 56, 8542. [CrossRef]

27. Baskes, M. Modified embedded-atom potentials for cubic materials and impurities. Phys. Rev. B 1992, 46, 2727. [CrossRef]

28. Kang, K.; Cai, W. Brittle and ductile fracture of semiconductor nanowires-molecular dynamics simulations. Phil. Mag. 2007, 87, 2169-2189. [CrossRef]

29. Plimpton, S. Fast parallel algorithms for short-range molecular dynamics. J. Comput. Phys. 1995, 117, 1-19. [CrossRef]

30. Li, J. AtomEye: An efficient atomistic configuration viewer. Model. Simul. Mater. Sci. Eng. 2003, 11, 173. [CrossRef]

31. Koh, S.; Lee, H.; Lu, C.; Cheng, Q. Molecular dynamics simulation of a solid platinum nanowire under uniaxial tensile strain: Temperature and strain-rate effects. Phys. Rev. B 2005, 72, 085414. [CrossRef]

32. Liu, Q.; Shen, S. On the large-strain plasticity of silicon nanowires: Effects of axial orientation and surface. Int. J. Plast. 2012, 38, 146-158. [CrossRef]

33. Swenson, R.J. Comments on virial theorems for bounded systems. Am. J. Phys. 1983, 51, 940-942. [CrossRef]

34. Subramaniyan, A.K.; Sun, C. Continuum interpretation of virial stress in molecular simulations. Int. J. Solids Struct. 2008, 45, 4340-4346. [CrossRef]

35. Jing, Y.; Meng, Q.; Gao, Y. Molecular dynamics simulation on the buckling behavior of silicon nanowires under uniaxial compression. Comput. Mater. Sci. 2009, 45, 321-326. [CrossRef]

36. Brantley, W. Calculated elastic constants for stress problems associated with semiconductor devices. J. Appl. Phys. 1973, 44, 534-535. [CrossRef]

37. Wang, Y.-C.; Xie, D.-G.; Ning, X.-H.; Shan, Z.-W. Thermal treatment-induced ductile-to-brittle transition of submicron-sized Si pillars fabricated by focused ion beam. Appl. Phys. Lett. 2015, 106, 081905. [CrossRef]

38. Kim, H.; Tomar, V. Nanometer to micron scale mechanics of [100] silicon nanowires using atomistic simulations at accelerated time steps. Phys. Status Solidi (A) 2011, 208, 2115-2123. [CrossRef] 
39. Kuo, J.-K.; Huang, P.-H.; Wu, W.-T.; Lu, C.-M. Mechanical and fracture behaviors of defective silicon nanowires: Combined effects of vacancy clusters, temperature, wire size, and shape. Appl. Phys. A 2014, 114, 1247-1256. [CrossRef]

40. Kizuka, T.; Takatani, Y.; Asaka, K.; Yoshizaki, R. Measurements of the atomistic mechanics of single crystalline silicon wires of nanometer width. Phys. Rev. B 2005, 72, 035333. [CrossRef]

41. Shimizu, F.; Ogata, S.; Li, J. Theory of shear banding in metallic glasses and molecular dynamics calculations. Mater. Trans. 2007, 48, 2923-2927. [CrossRef]

42. Steighner, M.; Snedeker, L.; Boyce, B.; Gall, K.; Miller, D.; Muhlstein, C. Dependence on diameter and growth direction of apparent strain to failure of Si nanowires. J. Appl. Phys. 2011, 109, 033503. [CrossRef]

43. Xu, S.; Su, Y.; Chen, D.; Li, L. An atomistic study of the deformation behavior of tungsten nanowires. Appl. Phys. A 2017, 123, 788. [CrossRef]

44. Diao, J.; Gall, K.; Dunn, M.L. Yield strength asymmetry in metal nanowires. Nano Lett. 2004, 4, $1863-1867$. [CrossRef]

45. Diao, J.; Gall, K.; Dunn, M.L. Surface-stress-induced phase transformation in metal nanowires. Nat. Mater. 2003, 2, 656. [CrossRef] [PubMed]

(C) 2019 by the authors. Licensee MDPI, Basel, Switzerland. This article is an open access article distributed under the terms and conditions of the Creative Commons Attribution (CC BY) license (http://creativecommons.org/licenses/by/4.0/). 